\title{
Influence of Different Modeling Strategies for CFRP on Finite Element Simulation Results
}

\author{
Xueshu LIU a, Li HUANG, Maoqing XU, Zhipeng ZHANG \\ School of Automotive Engineering, Dalian University of Technology, \\ Dalian 116024, P.R. China \\ ${ }^{a}$ liuxs@dlut.edu.cn ${ }^{1}$
}

\begin{abstract}
Numerical simulation is used to predict the behavior and response of carbon fiber reinforced plastic (CFRP). Sometimes zero thickness of interface layer is introduced into the numerical model to investigate the inter-layer behavior like delamination. To investigate the influence of critical volume-type defect like void, usually appeared in matrix rich region at the interface between layers, on mechanical properties of CFRP, numerical models with different interface thickness were created and tensile property and three-point bending simulation results were compared to experimental ones. It is found that accurate result is obtained with increasing of the interface thickness and up to $20 \%$ that of layer thickness is recommended to model the matrix rich region.
\end{abstract}

\section{Introduction}

Carbon fiber reinforced plastic (CFRP) is light weight and high specific stiffness and strength, all of which make it suitable for a wide range of high responsibility applications from spacecraft to sports equipments to satellite dishes. CFRP component is a typical laminated structure (Fig.1 a) and each layer in laminate is named lamina made of matrix and unidirectional fibers (Fig.1 b). Laminae can be fabricated in different fiber orientation and thickness for excellent macroscopic mechanical properties of the laminate. But laminae usually share the same thickness in real application such as the structure made by prepreg tapes.

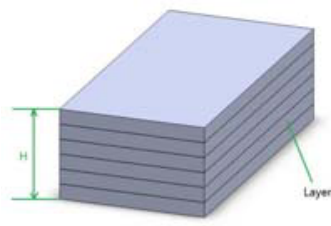

(a) Laminate

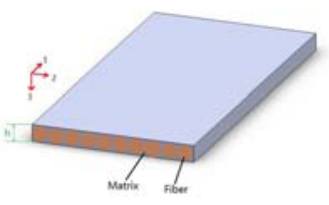

(b) Lamina

Fig. 1 CFRP composition

* Corresponding author:liuxs@dlut.edu.cn 
The macroscopic properties of lamina are important for design because only with them the potential performance of a laminate is predictable after taking the lay-up sequence into consideration. Although experiments are the most reliable way, analytical approach and numerical simulation are usually preferred when time and expense are considered. So, many methods have been proposed for predicting the elastic constants of a lamina, among which the rule of mixture approach is the simplest one. Following equations are used for the elastic constants approximation [1].

$$
\begin{aligned}
& E_{1}=E_{f} V_{f}+E_{m}\left(1-V_{f}\right) . \\
& E_{2}=\frac{E_{m}\left(E_{f}-E_{m}\right) V_{f}}{E_{m} V_{f}^{1}+E_{f}\left(V_{f}^{I}\right)}+E_{m} . \\
& G_{12}=\frac{G_{m}\left(G_{f}-G_{m}\right) V_{f}}{G_{m} V_{f}^{I}+G_{f}\left(V_{f}^{I}\right)}+G_{m} . \\
& G_{23}=\frac{E 2}{2\left(1+v_{23}\right)} . \\
& v_{12}=v_{f} V_{f}+v_{m}\left(1-V_{f}\right) . \\
& v_{23}=\frac{v_{12}}{1-v_{12}}\left(1-v_{12} \frac{E_{2}}{E_{1}}\right) .
\end{aligned}
$$

where $E_{1}$ and $v_{12}$ indicate the longitudinal elastic modulus and Poisson's ratio, $E_{2}$ is the transverse elastic modulus, $\mathrm{G}_{\mathrm{f}}$ and $\mathrm{G}_{\mathrm{m}}$ are the shear modulus of fiber and matrix, $v_{\mathrm{f}}$ and $v_{\mathrm{m}}$ signify the Poisson's ratio of fiber and matrix and $V_{f}$ is the volume fraction of fiber in composite.

In practice CFRP is always regarded as a transversely isotropic material for the sake of simplicity. Therefore, $E_{2}=E_{3}, G_{12}=G_{13}$ and $v_{12}=v_{13}$ are hold. Given these elastic constants it becomes possible to predict the mechanical performance of CFRP structure by using numerical simulation.

Note that the mentioned approach for lamina's properties approximation is based on some hypotheses including the composite material response is in direct relation with the volume fractions of compounding materials, namely fiber and matrix, and homogeneous distribution of phases is considered in the composite. So all laminae have the same mechanical properties and the response of laminate mainly depends on the layup sequence of the laminate.

However, it is obvious that macroscopic properties of composite material are strongly influenced and determined by the properties of the micro-constituents. Therefore, to guaranty the mechanical performance of a structure it is essential to avoid any defect in the composite material during the manufacturing process. However, challenges are still remaining to make CFRP achieve the desired high performance. The manufacturing route of CFRP is complex due to the fact that many control parameters are involved and defects like void, contamination and delamination have many chances to be introduced into the material and their appearances consequently lower the mechanical performance of CFRP component [2-6]. To take the influence of these potential defects into consideration, reducing the macroscopic properties of lamina is a general way. But it can not be used to identify the local behaviors and critical micro-structural features such as to investigate the effect critical void on the mechanical performance of a structure by numerical simulation. 
The purpose of this paper is to investigate the influence of different modeling strategies for CFRP on finite element simulation results and propose a modeling method for CFRP materials, which could be applied to evaluate the influences of critical micro-structural features on the mechanical properties of lamina like void.

Experiments. For the validation of numerical simulation results two type of experiments, tensile property test and three-point bending test, are carried out and the details are introduced in this section.

All specimens used for tests are manufactured by CFRP prepreg tapes IMS194/CYCOM 977-2 with symmetrical layup sequence $[45 / 90 /-45 / 0 / 90 / 0 /-45 / 90 / 45 /-45]_{\text {s }}$. The fiber volume fraction of the material is $57.61 \%$, the nominal thickness of a lamina is $0.188 \mathrm{~mm}$ and its mechanical properties are given in Table 1 . There are 20 layers used for each specimen and the nominal thickness is $3.76 \mathrm{~mm}$.

TABLE 1 ELASTIC CONSTANTS OF IMS194/CYCOM977-2 LAMINA

\begin{tabular}{cccccc}
\hline$E_{1}(\mathrm{GPa})$ & $\begin{array}{c}E_{2} \\
(\mathrm{GPa})\end{array}$ & $G_{12}(\mathrm{GPa})$ & $G_{23}(\mathrm{GPa})$ & $v 12$ & $v 23$ \\
\hline 165 & 8.20 & 2.80 & 2.77 & 0.33 & 0.48 \\
\hline
\end{tabular}

With Equ.1-6 and Table1, the properties of fiber and matrix are obtained: $E_{\mathrm{f}}=284 \mathrm{GPa}$, $\mathrm{E}_{\mathrm{m}}=3.52 \mathrm{GPa}, \mathrm{G}_{\mathrm{f}}=116 \mathrm{GPa}, \mathrm{G}_{\mathrm{m}}=1.20 \mathrm{GPa}, v_{\mathrm{f}}=0.22$ and $v_{\mathrm{m}}=0.48$.

Experiments were prepared and tested according to ASTM D5961/D5961M-13 and ASTM D3039/D3039M-08 standard. All tests were conducted on a WDW-100 electronic universal testing machine with a load capacity of $100 \mathrm{kN}$ as shown in Fig.2.

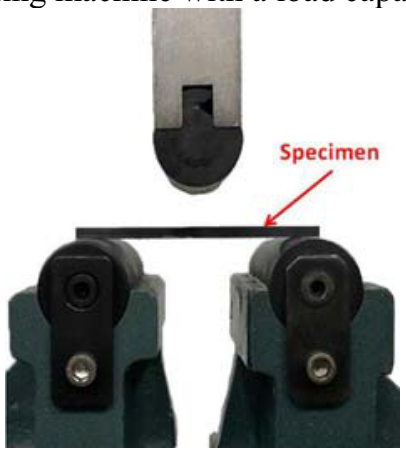

(a) Tensile property test

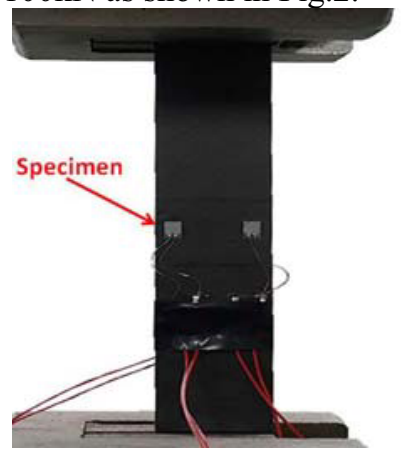

(b) Three-point bending test

Fig. 2 Tests: tensile property test and three-point bending test

Five specimens were prepared for each test and the averaged results were used to evaluate the numerical simulation. Note that only data in linear elastic stage are used for numerical model evaluation.

\section{Modeling Method}

As mentioned above, lamina is always regarded as a composite, a perfect mixture of fiber and matrix, in numerical simulation (Fig.1 a). But as a matter of fact the interlayer region is of matrix rich for prepreg tape made component and defect such as void is likely to appear there (Fig.3). Therefore, this region should be considered in numerical model, especially when the effects of voids on mechanical performance of structure are concerned. 


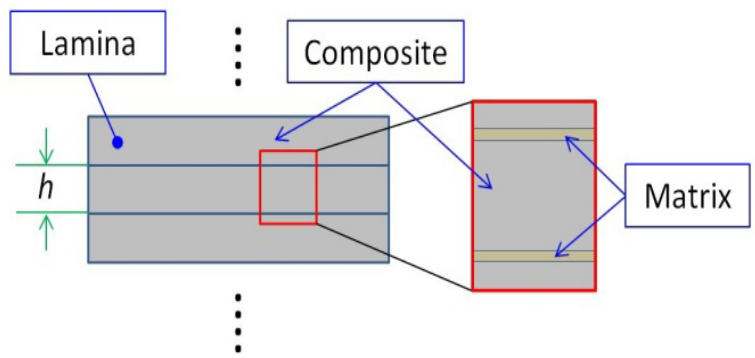

Fig. 3 Modeling methods: matrix rich region is considered in this study.

In many numerical studies the interface (matrix rich region) is treated as zero thickness to model delamination propagation $[7,8,9]$. But for the volume-type defects like voids it is impossible for them to appear in zero thickness layer because there is no room for accommodation. To investigate the influence of interface thickness on simulation results, serial numerical models with different interface thickness have been created, which are based on the following hypotheses:

1) Lamina is modeled as sandwich structure: a composite core (perfect mixture of fiber and matrix) and two matrix surface layers as shown in Fig.4. The two matrix layers have the same thickness $\delta$.

2) The thickness of lamina $\mathrm{h}$ is preserved in all models, that is, with the increasing of $\delta$ the thickness of the CFRP core decreases.

3) The fiber volume fraction $V_{f}$ of the core increases as $\delta$ increases but no more than $78.5 \%$, the maximum theoretical fiber volume fraction for idealized square fiberpacking geometry, which determines the span of $\delta[10]$.

4) As the serial/parallel mixing theory is hold in this study the mechanical properties of the core vary according to $\mathrm{V}_{\mathrm{f}}$.

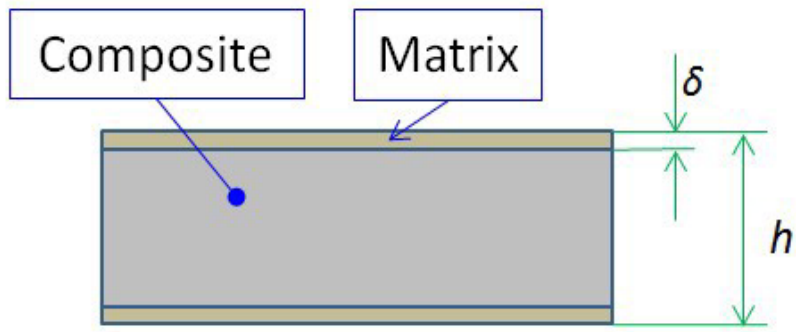

Fig. 4 Proposed lamina structure

Based on the above considerations the mechanical properties of the core in different numerical models are given in Table 2. Note that the first row is the given properties of lamina as shown in Table 1.

With the serial numerical models simulations were carried out and results were compared to experimental ones which are shown in details in the next section. 
TABLE 2 MECHANICAL PROPERTIES OF THE CFRP CORE IN DIFFERENT NUMERICAL MODELS

\begin{tabular}{cccccccc}
\hline $\begin{array}{c}\delta \\
(\mathrm{mm})\end{array}$ & $\begin{array}{c}V f \\
(\%)\end{array}$ & $\begin{array}{c}E_{1} \\
(\mathrm{GPa})\end{array}$ & $\begin{array}{c}E_{2} \\
(\mathrm{GPa})\end{array}$ & $\begin{array}{c}G 12 \\
(\mathrm{GPa})\end{array}$ & $\begin{array}{c}G 23 \\
(\mathrm{GPa})\end{array}$ & $v 12$ & $v 23$ \\
\hline 0 & 57.61 & 165 & 8.20 & 2.80 & 2.77 & 0.33 & 0.48 \\
0.01 & 60.85 & 174 & 8.55 & 3.00 & 2.92 & 0.32 & 0.46 \\
0.02 & 64.47 & 184 & 9.03 & 3.28 & 3.13 & 0.31 & 0.44 \\
0.03 & 68.55 & 195 & 9.72 & 3.45 & 3.42 & 0.30 & 0.42 \\
0.04 & 73.18 & 208 & 10.74 & 3.70 & 3.78 & 0.29 & 0.40 \\
0.05 & 78.48 & 223 & 12.42 & 4.22 & 4.22 & 0.28 & 0.38 \\
\hline
\end{tabular}

\section{Results and Discussion}

Simulation and experimental results of tensile property are shown in Fig.5. Fig.5 (a) shows the comparison of simulation results to test. Fig.5 (b) presents the details. It can be seen that the simulation results show good consistency in experiment and with the increasing of $\delta$ the simulation result becomes better but the trend is no linear which can be seen in Fig. 6 . The best result is obtained when $\delta=0.03 \mathrm{~mm}$, that is, $15.9 \%$ of the lamina thickness $h$.

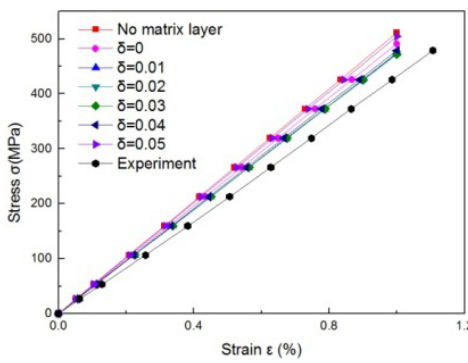

(a) Tensile results

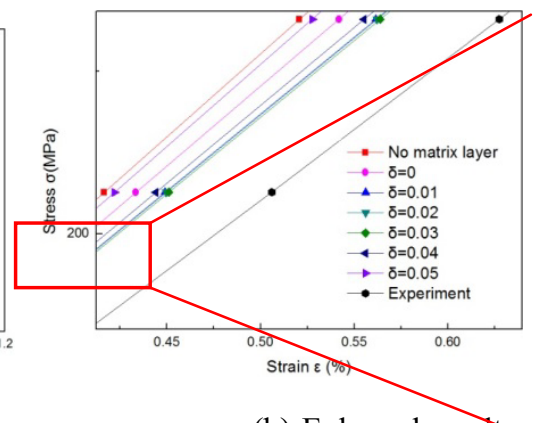

(b) Enlarged results

Fig. 5 Tensile results comparison

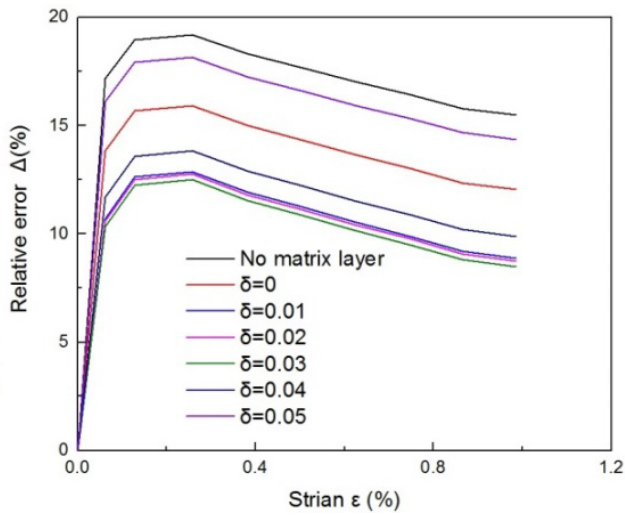

Fig. 6 Relative error of simulation results on tensile study 
Three-point bending of simulation and experiment results are shown in Fig.7. The linear performance of the material is perfectly reflected with all numerical models. The relative errors of simulation results to experiment are shown in Fig.8. When the strain is less than $1.3 \%$, best result is obtained when $\delta=0.04 \mathrm{~mm}$, namely $21.2 \%$ of $h$.

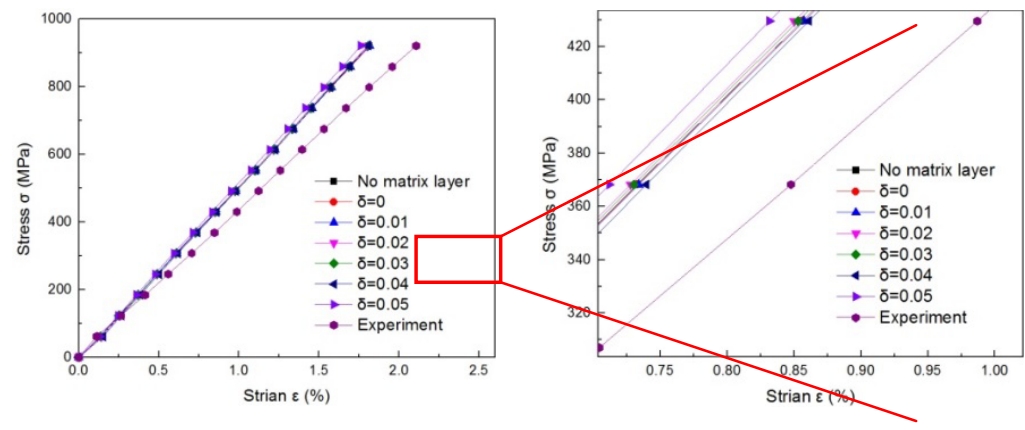

(a) Bending results

(b) Enlarged results

Fig. 7 Three-point bending results comparison

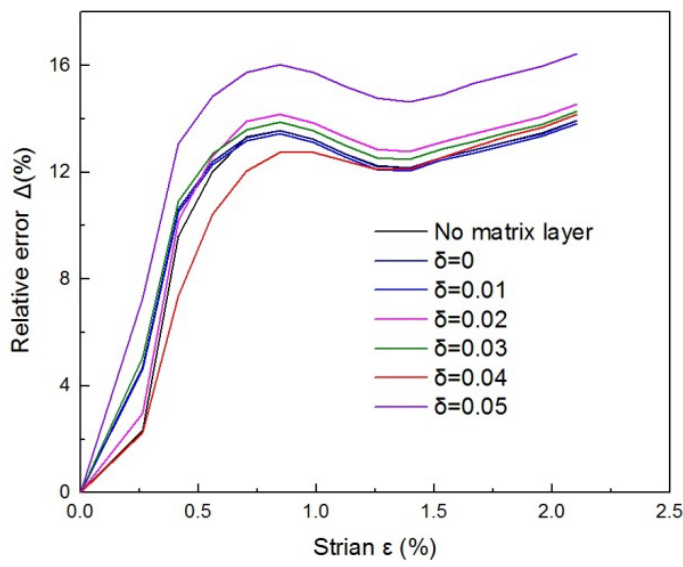

Fig. 8 Relative error of simulation results on three-point bending study

From all results, although $\delta$ affects the performance of composite, the influence is of limitation and the maximum difference is not larger than $8 \%$ compared to zero thickness, which means the macroscopic performance of laminate is not significantly affected by the thickness of interface layer. So the modeling method to thicken the matrix rich region so that the volume-type defect could be accommodated is acceptable. After taking fiber volume fraction into consideration $\delta=(0.15 \sim 0.2) \times h$ is recommended, where $h$ indicates the nominal thickness of a lamina as mentioned above.

\section{Conclusions}

In this paper, a modeling method for CFRP is proposed by considering the matrix rich region between layers. Serial numerical models were created and simulation results were compared to experiments. It was found that introducing the matrix rich region into numerical model is acceptable. The thickness of this layer has effect on simulation result 
but very limitation. A better result can be obtained by increasing the thickness of matrix rich region. Based on this study, 15-20\% of thickness of lamina is recommended for better simulation result. In addition, this study shows a way to investigate the effect of critical volume-type defect appeared in matrix rich region on mechanical performance of CFRP.

\section{Acknowledgements}

This work was supported by the National Natural Science Foundation of China [grant number 51375068, 51475073] and the Fundamental Research Funds for the Central Universities [grant number DUT14QY22] and the Scientific Research Foundation for the Returned Overseas Chinese Scholars, State Education Ministry. The authors would like to acknowledge the above financial supports.

\section{References}

1. R.M. Christensen. Tensor transformation and failure criteria for analysis of fiber composite materials. Journal of Composite Materials, 22:874-897, 1988.

2. K.D. Potter, M. Campbell, C. Langer, and M.R.Wisnom. The generation of geometrical deformations due to tool/part interaction in the manufacture of composite components. Composites: Part A, 36:301-308, 2005.

3. K. Potter, C. Langer, B. Hodgkiss, and S. Lamb. Sources of variability in uncured aerospace grade unidirectional carbon fibre epoxy preimpregnate. Composites: Part A, 38:905-916, 2007.

4. K. Potter, B. Khan, M. Wisnom, T. Bell, and J. Stevens. Variability, fibre waviness and misalignment in the determination of the properties of composite materials and structures. Composites: Part A, 39:1343-1354, 2008.

5. K.D. Potter. Understanding the origins of defects and variability in composites manufacture. In Proceedings of 17th International Conference on Composite Materials, 2009.

6. T.S. Mesogitis, A.A. Skordos, and A.C. Long. Uncertainty in the manufacturing of fibrous thermosetting composites: A review. Composites: Part A, 57:67-75, 2014.

7. A. Atas and C. Soutis. Strength prediction of bolted joints in CFRP composite laminates using cohesive zone elements. Composites: Part B, 58:25-34, 2014.

8. A. Atas and C. Soutis. Application of cohesive zone elements in damage analysis of composites: Strength prediction of a single-bolted joint in CFRP laminates. International Journal of Non-Linear Mechanics, 66:96-104, 2014.

9. V.P. Nguyen, P. Kerfriden, and S.P.A. Bordas. Two- and three-dimensional isogeometric cohesive elements for com- posite delamination analysis. Composites: Part B, 60:193-212, 2014.

10. Y.X. Zhou and Z.M. Huang. A modified ultimate failure criterion and material degradation scheme in bridging model prediction for biaxial strength of laminates. Journal of Composite Materials, 42(20):2123-2141, 2008. 